\title{
Direct dark matter search using liquid noble gases
}

\section{Teresa Marrodán Undagoitia*}

Universität Zürich

E-mail: marrodan@physik.uzh.ch

Liquid noble gases have proven a great potential as detector medium for dark matter searches. Particles interacting in these media produce ionized and excited atoms that lead to prompt light emission and the production of free electrons. Either the pulse shape of the emitted light or the ratio between light and measured charge can be used to discriminate between different types of interactions. In this way, nuclear recoils from dark matter interactions can be separated from the main background contribution, namely electronic recoils from natural radioactivity. In this paper, the working principle and the different existing types of liquid noble gas detectors are summarized. In addition, an overview of current and future experiments is presented.

25th Texas Symposium on Relativistic Astrophysics

December 6-10, 2010

Heidelberg, Germany

\footnotetext{
* Speaker.
} 


\section{Introduction}

Indirect evidence for cold dark matter is well established from astronomical observations but the nature of this matter is still unknown. One very popular candidate is the Weakly Interacting Massive Particle (WIMP) as several theories beyond the standard model independly predict such a particle [1]. Direct detection experiments aim to register a recoiling nucleus after an elastic collision of a WIMP in the detector medium. Such a measurement would provide information on the interaction probability of WIMPs with ordinary matter and on the WIMP mass.

The recoiling nucleus would, however, deposit only few keV energy and the predicted rate is less than an interaction per day and $\mathrm{kg}$. The required detectors should therefore have a low energy threshold, a large mass and a very low background. As the aimed sensitivity in cross section increases, the requirements on the allowed backgrounds become stronger. Construction materials, for example, have to be selected to minimize both their gamma activity and neutron emission.

\section{Liquid noble gases}

During the last years, liquid noble gas detectors have proven that the above mentioned requirements can be achieved. Mainly liquid argon (LAr) and liquid xenon (LXe) are used but also some $R \& D$ activities are going on to test the feasibility of liquid neon ( $\mathrm{LNe})$ detectors. All these cryogenic liquids allow for large detector masses and homogeneous targets, and provide high scintillation yields. Noble gases are also transparent to their own scintillation light.

There are two main detector concepts. Single phase detectors (liquid) read out only the scintillation light produced by particle interactions and achieve background reduction using pulse shape discrimination (most effective in LAr) and/or fidutial volume cuts. Double phase detectors (liquid/gas) detect both the scintillation light and the charge (ionized electrons) produced in the interaction. The electrons are drifted to the gas phase and their signal is amplified either via proportional scintillation [2] or electron multiplication [3]. Double phase detectors are operated as a Time Projection Chamber (TPC) and reduce the background via charge/light ratio, fiducilization, and eventually pulse shape discrimination. Event position reconstruction can be performed either by using only the light pattern in the photosensors for single phase detectors or by adding the $z$ component from the drift time in a double phase TPC. In the latter mode, position resolutions in the order of few mm are achievable. Section 3 discusses electronic and nuclear recoil calibration of liquid noble gases which is a key issue to understand their sensitivity to WIMP interactions.

Among the noble gases argon is widely used due to its great discrimination power using the pulse shape technique [4]. In addition it is easily available, cheap, and is relatively simple to purify since most impurities freeze at its liquid temperature. However, its scintillation wavelength of $125 \mathrm{~nm}$ makes the light detection challenging and the presence of the radioactive isotope ${ }^{39} \mathrm{Ar}$ (beta emitter with $1 \mathrm{~Bq} / \mathrm{kg}$ natural abundance) sets strong requirements on the discrimination. Although xenon doesn't have such strong pulse shape discrimination and its purification is slighly more complex, it has important advantages. It has a high density of $3 \mathrm{~g} / \mathrm{cm}^{3}$, increasing the shielding power for background. Its scintillation light at $178 \mathrm{~nm}$ allows to use photomultipliers (PMTs) with quartz window, and no wavelength-shifter is required. Additionally, there is no radioactive isotope besides the $\beta \beta$ candidate ${ }^{136} \mathrm{Xe}$ which has so far not been observed. If low thresholds are reached, 
xenon has the highest integrated cross section for a $100 \mathrm{GeV} / \mathrm{c}^{2}$ WIMP. In the last years, the use of liquid neon has also been considered as it also contains no long-lived radioisotope, scintillates strongly in the deep ultraviolet and provides the possibility of pulse shape discrimination due to two distinct time constants in the light production. Table 1 shows a summary of some physical properties of the three liquids.

Table 1: Summary of the main properties of LNe, LAr and LXe.

\begin{tabular}{|c|c|c|c|}
\hline \hline & LNe & LAr & LXe \\
\hline \hline $\mathrm{Z}(\mathrm{A})$ & $10(20)$ & $18(40)$ & $54(131)$ \\
\hline Density $\left[\mathrm{g} / \mathrm{cm}^{3}\right]$ & 1.2 & 1.4 & 3.0 \\
\hline Scintillation $\lambda[\mathrm{nm}]$ & 78 & 125 & 178 \\
\hline Boiling point $[\mathrm{K}]$ at $1 \mathrm{~atm}$ & 27 & 87 & 165 \\
\hline Scintillation $[\gamma / \mathrm{keV}]$ & 7 & 40 & 46 \\
\hline Radioactive isotopes & none & ${ }^{39} \mathrm{Ar}\left(\beta^{-}\right) \tau=269 \mathrm{y}$ & ${ }^{136} \mathrm{Xe}(\beta \beta)$ \\
& & ${ }^{42} \mathrm{Ar}\left(\beta^{-}\right) \tau=32.9 \mathrm{y}$ & $\tau>1.1 \times 10^{22} \mathrm{y}[5]$ \\
\hline \hline
\end{tabular}

Nowadays there exist several liquid argon and liquid xenon experiments and already proposals for larger (tons to multi-tons in mass) detectors, also including liquid neon. A review of their status is given in section 4 .

\section{Calibration}

In general for each liquid noble gas detector one needs to characterize the signal region (nuclear recoils), the background region (electronic recoils), as well as the energy scales of both electronic and nuclear recoils. Most detectors are calibrated with radioactive sources from the outside. For example, ${ }^{137} \mathrm{Cs}$ or ${ }^{60} \mathrm{Co}$ have high energy lines that can be used to determine the electronic recoil region at low energies due to Compton scattering processes. To determine the signal region commonly a neutron source is used. However, as the detectors get larger (distances to the center in the order of tens of centimeters) the probability for single scatters of both gamma and neutron particles in the center decreases drastically. A proper calibration starts to be a challenging issue as detector size and sensitivity increase (order of one meter).

For the determination of the energy scale, the energy dependence of light/charge losses has to be known. These losses are usually called quenching.

\subsection{Electronic recoil calibration}

The electronic recoil energy scale and its linearity have to be calibrated specially in the low energy region relevant for dark matter searches $(\mathscr{O}(\mathrm{keV})$ or tens of $\mathrm{keV})$. Recently a novel calibration source, ${ }^{83 m} \mathrm{Kr}$ which is a decay product of ${ }^{83} \mathrm{Rb}$, has been tested in LXe both in single [6] and double phase [7] detectors and also in LAr and LNe [8]. The metastable state ${ }^{83 m} \mathrm{Kr}$ decays producing a $31.2 \mathrm{keV}$ line with a half-life of $1.83 \mathrm{~h}$ to the first ${ }^{83} \mathrm{Kr}$ excited state at $9.4 \mathrm{keV}$ which subsequently decays with a half-life of $154 \mathrm{~ns}$ to the ground state. This source could be introduced to the liquid target via the gas system, resulting in a homogeneous source distribution. The halflife 
is short enough to not disturb the dark matter search and its de-excitation lines are in the energy region of interest.

The measurements performed in liquid argon [8] show a constant behaviour of the light yield in the energy region from $40 \mathrm{keV}$ to $662 \mathrm{keV}$ at zero drift field. In liquid xenon, however, it has been seen [7] that the light yield increases with decreasing energy (down to $9.4 \mathrm{keV}$ ) at zero drift field. When a field is applied, the overall light yield decreases because the scintillation light from electron recombination is reduced. The relative increase of light yield at low energies is, however, larger than at zero field due to the energy dependence of the field quenching (decrease of field quenching for decreasing energy).

\subsection{Nuclear recoil calibration}

Dark matter detectors are calibrated for nuclear recoil interactions using neutron sources which produce a nuclear recoil spectrum up to tens of keVs. The nuclear recoil scale is usually calibrated in dedicated laboratory setups by performing neutron scattering experiments. In such experiments, a certain scattering angle is set corresponding to a fixed nuclear recoil energy which is know from kinematics. The light or charge signal resulting from the nuclear recoil can be compared to the signal from a known electronic recoil source to make the measurement independent of the detector in use. For this purpose, the $122 \mathrm{keV}$ line of ${ }^{57} \mathrm{Co}$ is widely used. All energy dependences of the nuclear recoil quenching are included in a variable called $L_{e f f}$.

Figure 1 shows all the current direct measurements of the nuclear recoil quenching for xenon (left) and argon (right). In the case of xenon, the three measurements at the lowest energies differ
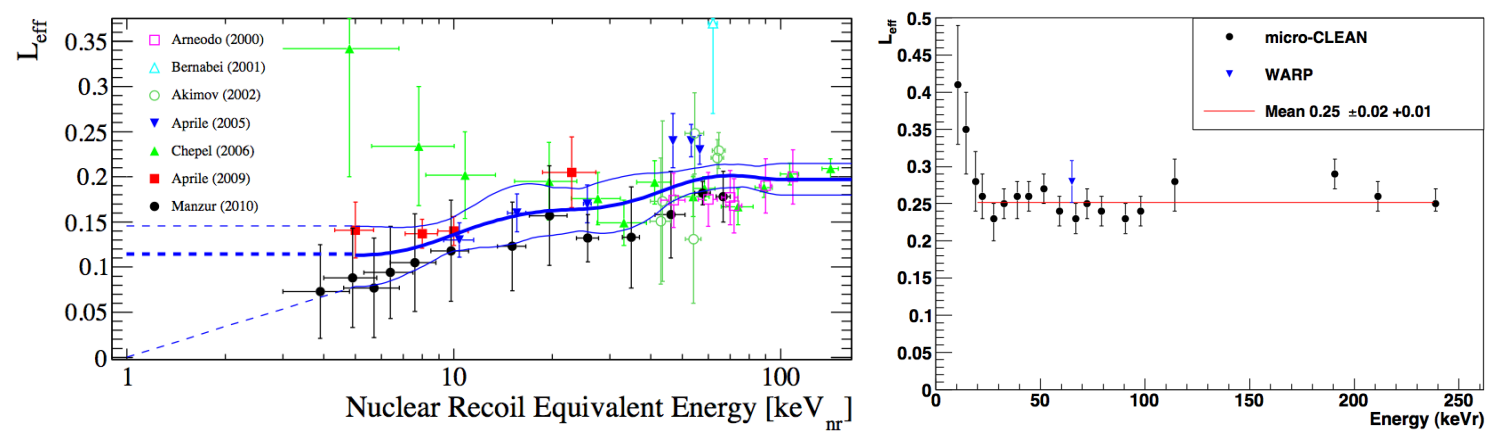

Figure 1: Energy dependence of the nuclear recoil quenching. The figure on the left shows $L_{e f f}$ for xenon from [9] and the figure on the right for argon from [10].

systematically from each other. The thick blue curve is a best fit to all these data, as used by the XENON100 collaboration [9] to set their nuclear recoil scale. New measurements are ongoing [11] to clearify the situation but also discussions [12] on the possible origin of the discrepancies. For argon there are so far only two measurements existing, compatible with each other. Commonly energy thresholds of 30-40 keV are achieved in dark matter experiments using argon. Above this threshold a constant behaviour of the nuclear quenching can be assumed, however based on few experimental points only. 


\section{Current experimental status}

The use of liquid noble gases for dark matter detection has spread in the last years. This section describes very briefly past and present experiments, and gives an overview of the projects in construction or in planning phase.

\subsection{Liquid argon detectors}

The first liquid argon detector delivering dark matter results was the WARP [13] experiment, a $2.3 \ell$ double phase TPC located at LNGS (Laboratori Nationali del Gran Sasso) in Italy. The detector was read out with two arrays of photosensors. It achieved a sensitivity in cross section of $\sigma<10^{-42} \mathrm{~cm}^{2}$ due to an excellent background rejection of $3 \times 10^{7}$ at $50 \%$ nuclear recoil acceptance and 50 pe energy threshold. This was due to a combination of pulse shape and charge to light ratio as discrimination parameters. After the success of this first detector, the collaboration designed and constructed the larger detector WARP- $140 \mathrm{~kg}$ which is currently in commissioning phase also at LNGS.

The ArDM [14] detector can be considered as the first ton-scale liquid argon experiment. It is also a double phase TPC containing $850 \mathrm{~kg}$ target volume which has been constructed and tested at CERN. Eight PMTs on the bottom detect the scintillation light. The charges are drifted over a maximum drift length of $120 \mathrm{~cm}$ to the planned read out on top. A drift field of $1-4 \mathrm{kV} / \mathrm{cm}$ is planned. The main technical difference of this detector is that the charge read out is done directly in Large Electron Multipliers (LEMs [3]) and that the HV for the drift field is generated inside the detector. Currently the collaboration is preparing to move the detector to the Canfranc underground laboratory in Spain.

There are two further liquid argon experiments under construction at SNOlab (in Canada): DEAP [15] and CLEAN [16]. The MiniCLEAN experiment [17] is a single phase detector of $150 \mathrm{~kg}$ fiducial mass which is designed to be filled with argon and later with neon. It has a spherical geometry and $4 \pi$ coverage by light guides that bring the scintillation light to the PMTs. Pulse shape discrimination will be used to reduce the backgrounds. Currently the detector is under construction. The DEAP experiment foresees a mass of $3600 \mathrm{~kg}$ of liquid argon in single phase. It aims to use depleted argon to reduce the background from ${ }^{39} \mathrm{Ar}$. The detector is also in construction phase and its assembly is planned for 2012 .

\subsection{Liquid xenon detectors}

After running two previous prototypes, the ZEPLIN collaboration is currently operating the ZEPLIN-III detector [18] at the Boulby mine in the UK. The detector consists of a flat TPC of $30 \mathrm{~cm}$ diameter and $3.6 \mathrm{~cm}$ drift height. In a first run with an exposure of $268 \mathrm{~kg} \cdot \mathrm{d}, 7$ events were found in the region of interest. The background prediction was $11 \pm 3$ events. This result allowed the collaboration to place a limit in the WIMP-nucleon cross section of $8.1 \times 10^{-8} \mathrm{pb}$ for a $60 \mathrm{GeV} / c^{2}$ WIMP mass at $90 \%$ C.L. Last year, several detector upgrades were performed (for example new PMTs, new calibration system) and a new dark matter run was started.

The XENON10 [19] detector was a $15 \mathrm{~kg}$ double phase detector which was running at LNGS in 2007. Recently, the dark matter data from this detector has been reanalyzed using the charge signal (S2) as the only energy scale [20]. As the S2 signal is amplified, a lower energy threshold 
can be achieved. This leads to an improved sensitivity to low-mass WIMPs which produce low energy nuclear recoils. The XENON100 detector [9] installed at the same location as XENON10, is currently one of the leading experiments in the dark matter field. It is a double phase TPC of $62 \mathrm{~kg}$ target mass. Due to its extremely low electronic recoil background of less than $10^{-2}$ events $\cdot \mathrm{kg}^{-1} \cdot \mathrm{d}^{-1} \cdot \mathrm{keV}^{-1}$ [21] it has a great sensitivity for WIMP detection. Recently, first results from the analysis of $11.2 \mathrm{~d}$ data taken during a commissioning run have been released setting a best cross section limit of $3.4 \times 10^{-44} \mathrm{~cm}^{2}$ at $55 \mathrm{GeV} / \mathrm{c}^{2}$ WIMP mass. Since then, the detector has collected about $100 \mathrm{~d}$ of blinded data and the results are expected within 2011.

In the last year, the construction of the XMASS detector [23] at the Kamioka mine in Japan has been completed. XMASS is a $800 \mathrm{~kg}$ single phase xenon detector which has been designed to reach very low backgrounds (at the level of $10^{-4}$ events $\cdot \mathrm{kg}^{-1} \cdot \mathrm{d}^{-1} \cdot \mathrm{keV}^{-1}$ ). This is achieved with a water shield of $\sim 800$ ton and the very low radioactivity materials used in its construction. Currently, it is in commissioning phase and the dark matter run is planned to start within 2011. Besides the search for dark matter, the XMASS experiment aims to measure solar neutrinos via their scattering on electrons and the neutrinoless double beta decay of the candidate isotope ${ }^{136} \mathrm{Xe}$.

In the US, the LUX collaboration [24] has constructed a $350 \mathrm{~kg}$ two phase TPC. The design is similar to the XENON detector. Light and proportional scintillation light from the charge signal are read with two arrays of 2 inch PMTs. The experiment aims for a background level lower than $10^{-3}$ events $\cdot \mathrm{kg}^{-1} \cdot \mathrm{d}^{-1} \cdot \mathrm{keV}^{-1}$ by using a water shield and low radioactive construction materials. Currently, the detector is being tested above ground at the Sandford surface facility (US) and will be moved to the Homestake mine as soon as the underground infrastructure is ready.

\subsection{R\&D activities and future plans}

The DarkSide detector has been proposed recently, a $50 \mathrm{~kg}$ depleted argon detector which could be placed inside the Counting Test Facility (CTF) of Borexino [25]. The organic liquid scintillator which fills the CTF detector would be loaded with ${ }^{10} \mathrm{~B}$ and used as neutron veto to reduce this background.

The XENON collaboration has started the design of a larger detector, XENON1T [22]. The experiment will use a target mass of about 2 tons ( 1 ton fiducial, 2.4 ton total mass) and aims for a total background rate 100 times lower than in XENON100.

In addition, there are multi-ton scale future projects under study both in Europe and in the US: DARWIN [26] and MAX [27], respectively. These projects aim for multi-ton scale LAr and LXe dark matter search facilities, with the goal of probing the cross section region below $10^{-47} \mathrm{~cm}^{2}$, or to provide a high-statistics measurement of WIMP interactions in case of a positive detection by one of the running experiments.

\section{Acknowledgments}

Thanks to the conference organizers for the invitation. M. Schumann and Q. Weitzel are acknowledged for their comments to this document. 


\section{References}

[1] G. Bertone, D. Hooper, and J. Silk, Phys. Rep. 405, 279 (2005)

[2] A. Lansiart et al., Nucl. Instrum. Methods 135, 47 (1976)

[3] A. Bondar et al., JINST 3 (2008) 07001

[4] W. H. Lippincott et al., Phys. Rev. C 78, 035801 (2008), arXiv:0801.1531

[5] R. Bernabei et al., Nucl. Phys. B (Proc. Suppl.) 11088 (2002)

[6] L. W. Kastens et al., Phys. Rev. C 80, 045809 (2009), arXiv:0905.1766

[7] A. Manalaysay et al., Rev. Sci. Instrum. 81, 073303 (2010), arXiv:0908.0616

[8] W. H. Lippincott et al., Phys. Rev. C 81, 045803, (2010), arXiv:0911.5453

[9] E. Aprile et al., (XENON100 Collaboration), Phys. Rev. Lett. 105, 131302 (2010)

[10] D. Gastler et al., arXiv:1004.0373

[11] G. Plante, talk at PCTS Workshop "Dark Matter: Direct Detection and Theoretical Developments"

[12] A. Manalaysay, arXiv:1007.3746

[13] R. Brunetti et al. (WARP Collaboration), Astropart. Phys. 28, 495-507 (2008)

[14] A. Rubbia et al. (ArDM Collaboration), J. Phys. Conf. Ser. 39, 129 (2006)

[15] M. G. Boulay et al., arXiv:0904.2930

[16] D. N. McKinsey and K. J. Coakley, Astrop. Phys. 22 (2005) 355

[17] D. N. McKinsey et al., (Mini-CLEAN Collaboration), Nucl. Phys. B (Proc. Suppl.) 173 (2007) 152

[18] V. N. Lebedenko et al. (ZEPLIN-III Collaboration), Phys. Rev. D 80, 052010, (2009), V. N. Lebedenko et al. (ZEPLIN-III Collaboration), Phys. Rev. Lett. 103, 151302, (2009)

[19] J. Angle et al. (XENON10 Collaboration), Phys. Rev. Lett. 100, 021303 (2008), J. Angle et al. (XENON10 Collaboration), Phys. Rev. Lett. 101, 091301 (2008)

[20] P. Sorensen (for the XENON10 Collaboration), talk at the IDM conference (Montpellier 2010)

[21] E. Aprile et al., (XENON100 Collaboration), arXiv:1101.3866

[22] E. Aprile et al. (XENON1T Collaboration), XENON1T at LNGS, Proposal, April (2010)

[23] K. Abe et al., (XMASS Collaboration), J. Phys. Conf. Ser. 120 (2008) 042022

[24] LUX Collaboration, http://lux.brown.edu/index.html

[25] G. Ranucci et al. (Borexino Collaboration), Nucl. Phys. Proc. Suppl. 70, 377 (1999)

[26] L. Baudis et al., DARWIN Consortium, PoS IDM2010 122 (2010), arXiv: 1012.4767

[27] http://www.fnal.gov/pub/max/ 